\title{
Type 2 Diabetes Mellitus, Insulin Resistance, Obesity, there is Any Association with Liver Cancer?
}

\author{
Ibrahim El Bayoumy* \\ Department of Public Health and Community Medicine, Tanta Faculty of Medicine, Egypt \\ *Corresponding author: Ibrahim El Bayoumy, Professor of public health and community medicine, Tanta faculty of medicine, Egypt
}

Submission: 眥August 13, 2018 ; Published: 眥August 14, 2018

\section{Introduction}

Hepatocellular carcinoma (HCC) accounts for $70-85 \%$ of primary liver cancers, ranking fifth among the most common malignancies worldwide and third as a cause of cancer death. Dominant etiological factors of HCC show great variations according to different geographic areas. In Asia, vertically transmitted hepatitis. B infection is a major contributor to HCC, which may emerge in non-cirrhotic livers due to direct oncogenic properties of the hepatitis B virus. In sub-Saharan Africa, dietary exposure to aflatoxin may synergize with the carcinogenic effects of chronic hepatitis B infection. In the Western world, HCC most often complicates cirrhosis evolving from chronic viral hepatitis B and C, alcoholic injury, or inherited disorders such as hemochromatosis $[1,2]$.

\section{Liver Cancer and Diabetes}

There is now a large body of evidence indicating a strong and consistent increased risk of incident cancer associated with diabetes [3]. For type 2 diabetes, the strength of the association depends on the specific cancer site, with the strongest relationships observed for liver and pancreatic cancers, followed by endometrial, postmenopausal breast, colorectal, bladder, non-Hodgkin's lymphoma, and kidney cancer [4]. For stomach cancer, there was an increased risk in a Japanese population [5], but it is not known if this extends to other populations, most of which have a much lower incidence of stomach cancer. The literature also consistently demonstrates a $10-20 \%$ decreased risk of prostate cancer among men with diabetes, due, in part, to the reduced levels of circulating testosterone levels in these individuals [6].

For other, rarer malignancies, the number of studies is small, and more importantly, they usually lack adequate power to reliably explore these associations. Similarly, for studies of cancer mortality, positive associations have been shown for cancers of the pancreas, liver, colon and rectum, and bladder [7]. However, there is little data for mortality of rare cancer outcomes. For type 1 diabetes, evidence is limited and variable. Cohort studies have shown a $10-37 \%$ increased risk for the incidence of all cancers combined, whereas case-control studies showed no association [8]. Studies are rarely large enough to explore site-specific cancer incidence. However, there is evidence to suggest an increased risk for cancers of the pancreas, liver, and stomach. The evidence of cancer-specific mortality among type 1 diabetic cohorts is even more limited.

\section{Possible Mechanisms}

The higher risk of HCC among people with diabetes could potentially be related to exposure of the liver to high insulin concentrations in the portal circulation that are particularly elevated among people with insulin resistance and type 2 diabetes, particularly if the tumor cells remain sensitive to insulin. Hyperinsulinemia could increase risk of HCC by increasing synthesis of insulin-like growth factor-1 (IGF-1) which promotes cell growth and proliferation and inhibits apoptosis with evidence for this mechanism provided by in vitro studies, animal models, and epidemiologic studies (reviewed in reference: A further possible biological mechanism for the association between diabetes and liver cancer is that up to $80 \%$ of people with type 2 diabetes are thought to have non-alcoholic fatty liver disease which increases risk of non-alcoholic steato-hepatitis, cirrhosis and subsequent HCC.

Experimental and clinical observations on the ability of antidiabetic medications to alter cancer risk by improving insulin resistance are accumulating [9]. Metformin, an activator of AMPactivated protein kinase, and thiazolidinediones, agonists of peroxisome proliferator-activated receptor may effectively reduce circulating glucose and insulin levels and limit their systemic effects on oncogenic pathways. In addition, metformin and thiazolidinediones reduce the extent of hepatic lipid accumulation, further limiting the organ-specific molecular events that may contribute to hepatocarcinogenesis [10]. The observations of Lai et al. [11] provide valuable additional evidence that properly controlled glucose and lipid homeostasis may help avoid or delay diabetes-associated complications, an unfortunately long list that

In conclusion, old age, low serum TG levels and high serum GGT levels may predict risk of HCC in diabetic patients who do not have chronic viral hepatitis or alcoholic cirrhosis. Scoring system 
using these parameters may be useful in identifying subgroups of the diabetic population who are at an increased risk of HCC. In clinical practice, clinicians are increasingly required to manage and treat patients with both T2DM and HCC. Although there are still important gaps in our knowledge(s), the use of metformin may be associated with a lower incidence of HCC. To date, studies reporting on the effect of glucose-lowering medications other than metformin on HCC prognosis are both scant and difficult to interpret, owing to the complexity of pharmacotherapy for T2DM, and the many sources of bias that this complexity may generate. Further research is required to clarify the variables that contribute to the complexity of the associations between T2DM, hyperglycemia, diabetes treatment and HCC risk.

\section{References}

1. Bosch FX, Ribes J, Cleries R, Díaz M, et al. (2005) Epidemiology of heptocellular carcinoma. Clin Liver Dis 9(2): 191-211, v.

2. El-Serag HB, Rudolph KL (2007) Hepatocellular carcinoma: epidemiology and molecular carcinogenesis. Gastroenterology 132(7): 2557-2576.

3. Giovannucci E, Harlan DM, Archer MC, Bergenstal RM, Gapstur SM, et al. (2010) Diabetes and cancer: a consensus report. Diabetes Care 33(7): 1674-1685.

4. Johnson JA, Carstensen B, Witte D, Bowker SL, Lipscombe L, et al. (2012) Diabetes and Cancer Research Consortium. Diabetes and cancer (1): evaluating the temporal relationship between type 2 diabetes and cancer incidence. Diabetologia 55(6):1607-1618.
5. Yamagata H, Kiyohara Y, Nakamura S, Kubo M, Tanizaki Y, et al. (2005) Impact of fasting plasma glucose levels on gastric cancer incidence in a general Japanese population: the Hisayama study. Diabetes Care 28(4): 789-794.

6. Kasper JS, Giovannucci E (2006) A meta-analysis of diabetes mellitus and the risk of prostate cancer. Cancer Epidemiol Biomarkers Prev 15(11): 2056-2062.

7. Renehan AG, Yeh HC, Johnson JA, Wild SH, Gale EA, et al. (2012) Diabetes and Cancer Research Consortium. Diabetes and cancer (2): evaluating the impact of diabetes on mortality in patients with cancer. Diabetologia 55(6): 1619-1632.

8. Gordon-Dseagu VLZ, Shelton N, Mindell JS (2013) Epidemiological evidence of a relationship between type- 1 diabetes mellitus and cancer: a review of the existing literature. Int J Cancer 132: 501-508

9. Giovanncci E, Harlan DM, Archer MC, Bergenstal RM, Gapstur SM, et al. (2010) Diabetes and cancer: a consensus report Diabetes care 33(7): 1674-1685.

10. Stickel F, Hellerbrand C (2010) Non-alcoholic fatty liver disease as arisk factor for hepatocellular carcinoma: mechanism and implications. Gut 59(10): 1303-1307.

11. Lai S, Chen PC, Liao KF, Muo CH, Lin CC, et al. (2012) Risk of hepatocellular carcinoma in diabetic patients and risk reduction associated with anti-diabetic therapy: a population-based cohort study. Am J Gastroenterol 107(1): 46-52.
Creative Commons Attribution 4.0 International License

For possible submissions Click Here
Submit Article



